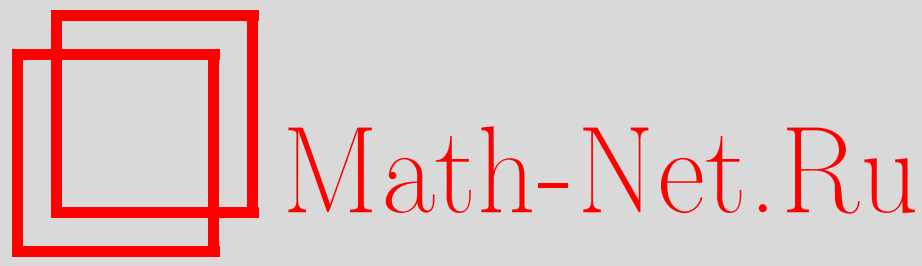

С. И. Доронин, Е. И. Кузнецова, Э. Б. Фельдман, Квантовый дискорд в трехспиновой системе во внешнем магнитном поле, ТМФ, 2014, том 181, номер 2, 387-400

DOI: https://doi.org/10.4213/tmf8676

Использование Общероссийского математического портала Math-Net.Ru подразумевает, что вы прочитали и согласны с пользовательским соглашением http://www . mathnet.ru/rus/agreement

Параметры загрузки:

IP : 3.85 .7 .115

26 апреля 2023 г., $15: 41: 54$

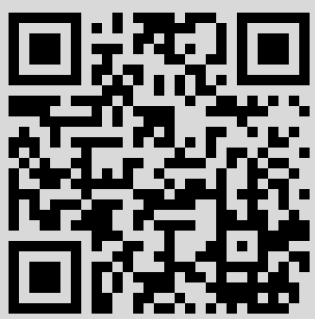




\title{
ФИЗИКА
}

Том 181, № 2

ноябрь, 2014

(C) 2014 г. С. И. Доронин*, Е. И. Кузнецова*, Э. Б. Фельдман*

\section{КВАНТОВЫЙ ДИСКОРД В ТРЕХСПИНОВОЙ СИСТЕМЕ ВО ВНЕШНЕМ МАГНИТНОМ ПОЛЕ}

\begin{abstract}
Изучены квантовые корреляции в двухсоставной гетероядерной $(2 \times 1)$-системе из трех спинов $S=1 / 2$ во внешнем магнитном поле как при высоких, так и при низких температурах. При высоких температурах минимум условной энтропии системы после проведения измерений на односпиновой подсистеме найден аналитически при любых соотношениях между частотами спинов обеих подсистем. В результате при высоких температурах впервые получены аналитические выражения для меры квантовых корреляций - квантового дискорда для трехкубитных систем. При низких температурах минимум условной энтропии найден численно. Исследована эволюция квантового дискорда и соотношение между классическими и квантовыми корреляциями.
\end{abstract}

Ключевые слова: квантовые корреляции, квантовый дискорд, квантовая условная энтропия, взаимная информация, редуцированная матрица плотности.

DOI: $10.4213 / \operatorname{tmf} 8676$

\section{1. ВВЕДЕНИЕ}

Квантовые корреляции, зависящие от измерений, расширяют многообразие корреляций в классических многочастичных системах и ответственны за многообещающие перспективы в развитии квантовых вычислений, криптографии, телепортации и метрологии [1]. Мерой квантовых корреляций служит квантовый дискорд [2], [3], который связан с неэквивалентностью различных определений квантовой условной энтропии [4] в квантовых системах. Будучи эквивалентными в классических системах, эти определения [4] ведут к разным результатам в квантовых системах из-за особенностей квантово-механической теории измерений [2], [3]. Соответствующие различия в квантовой условной энтропии могут поэтому рассматриваться как мера "квантовости" систем. В ряде случаев [5]-[7] удалось продемонстрировать, что квантовый дискорд является потенциальным ресурсом для обработки квантовой информации. Так, в модели детерминированных квантовых вычислений с одним кубитом (DQC1) [5], когда рассматривается один поляризованный кубит, связанный

* Институт проблем химической физики РАН, Черноголовка, Московская обл., Россия. E-mail: s.i.doronin@gmail.com 
с набором неполяризованных кубитов в максимально смешанном состоянии (квантовая запутанность в такой системе равна нулю), авторы работы [5] полагают, что вычислительная эффективность такой системы определяется квантовым дискордом. В экспериментах по квантовой метрологии [8], [9] удалось повысить точность измерений за счет квантовых корреляций, несмотря на то что запутанность в исследуемых системах отсутствовала. Считается [10], что результаты этих экспериментов также объясняются ненулевым дискордом.

Известно, что ядерный магнитный резонанс (ЯМР) используется для экспериментальной реализации квантовых алгоритмов в малокубитных системах. Для смешанных состояний, которые имеют место в жидкофазных ЯМР-экспериментах, запутанность при комнатных температурах обращается в ноль [11]. Отсюда возникли сомнения в возможностях ЯМР для демонстрации преимуществ квантовых алгоритмов над их классическими аналогами в многокубитных системах [1]. Квантовый дискорд в смешанных состояниях в ЯМР-экспериментах в отличие от запутанности не обращается в ноль [12], что свидетельствует о существовании в спиновой системе квантовых корреляций. Экспериментальные исследования [12], проведенные методами ЯМР-томографии [13], подтверждают этот вывод. В связи с изложенным разработка методов вычисления дискорда является весьма актуальной.

Вычисление квантового дискорда представляет собой достаточно трудоемкую задачу, поскольку оно требует проведения оптимизации квантовой условной энтропии по всем возможным полным наборам измерений на одной из подсистем рассматриваемой системы [2], [3]. Поэтому разработанные методы позволяют вычислить дискорд только в двухкубитных системах [14]-[16] и в простейших трехкубитных системах [17]. С целью упрощения вычислений в работах [18], [19] используется другая мера квантового дискорда - геометрический дискорд [20], [21]. Однако правомерность использования геометрического дискорда как меры квантовых корреляций до сих пор не выяснена [22]. В частности, величины квантового дискорда [2], [3] и геометрического дискорда [20], [21] могут существенно различаться [23]. Отметим также, что попытки определения квантового дискорда без использования процедуры оптимизации [24] нуждаются в обосновании.

В работе [25] мы предложили для вычисления квантового дискорда модель, в которой цепочка дипольно взаимодействующих спинов во внешнем магнитном поле связана с примесным спином гейзенберговским zz-взаимодействием. В этой модели квантовый дискорд может быть определен численно с помощью генетического алгоритма с мутациями [26]. Особое место в этой модели занимает трехспиновая система, в которой возможно аналитическое определение квантового дискорда. Этот случай может быть подкреплен экспериментальными исследованиями для гетероядерных систем [27], а также для электронно-ядерных систем со сверхтонким взаимодействием электронного и двух ядерных спинов [28]. Начатое в статье [25] исследование квантового дискорда в трехспиновых системах было ограничено случаем, когда ларморова частота примесного спина меньше ларморовой частоты остальных спинов. В настоящей работе это ограничение снимается и проводится полное аналитическое изучение квантового дискорда в рамках предложенной модели.

Статья организована следующим образом. В разделе 2 представлена трехспиновая модель для определения квантового дискорда. В разделе 3 в высокотемпера- 


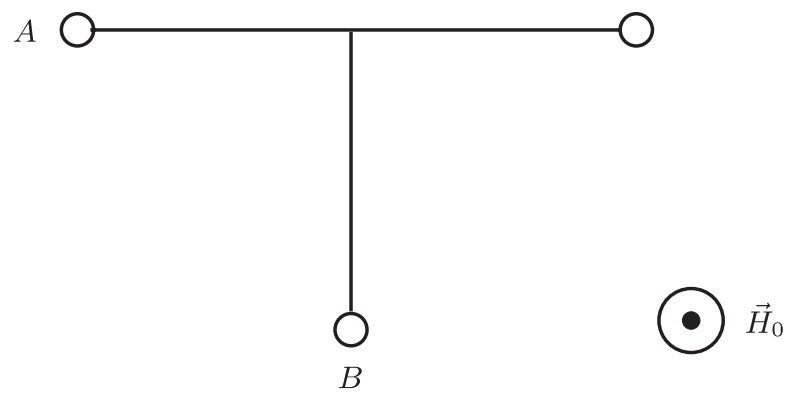

Рис. 1. Двухсоставная трехспиновая система для исследования квантовых корреляций. Магнитное поле $\vec{H}_{0}$ направлено перпендикулярно плоскости рисунка. Подсистема $A$ - пара спинов, связанных ДДВ. Примесный спин, связанный $z z$-взаимодействиями со спинами пары, представляет подсистему $B$.

турном приближении исследован минимум квантовой условной энтропии при различных соотношениях между ларморовскими частотами примесного спина и остальных спинов. Обсуждается физический смысл полученных результатов. В разделе 4 даны аналитические выражения для квантового дискорда при разных соотношениях между ларморовскими частотами и изучены квантовые корреляции при низких температурах. В заключении (раздел 5) подведены основные итоги проведенных исследований.

\section{2. ДВУХСОСТАВНАЯ ТРЕХСПИНОВАЯ МОДЕЛЬ ДЛЯ ИССЛЕДОВАНИЯ КВАНТОВЫХ КОРРЕЛЯЦИЙ}

Рассмотрим гетероядерную трехспиновую систему в сильном внешнем магнитном поле $\vec{H}_{0}$ (см. рис. 1$)$. Поле $\vec{H}_{0}$ направлено вдоль оси $z$, перпендикулярной плоскости рисунка. Два одинаковых спина $I=1 / 2$ связаны диполь-дипольным взаимодействием (ДДВ) (подсистема $A$ ), а третий спин другого сорта $S=1 / 2$ (подсистема $B$ ) равноудален от спинов подсистемы $A$ и связан с ними гетероядерным ДДВ [27].

Гамильтониан гетероядерных ДДВ может быть записан следующим образом:

$$
H=g I_{z} S_{z}
$$

где $g$ - константа ДДВ, $I_{z}=I_{1 z}+I_{2 z}$; здесь $I_{i \alpha}$ и $S_{\alpha}$ - проекции угловых спиновых моментов на ось $\alpha(i=1,2$ и $\alpha=x, y, z), \vec{x}, \vec{y}$ - оси во вращающейся системе координат (BCK), $\vec{x} \perp \vec{z}, \vec{y} \perp \vec{z}, \vec{x} \perp \vec{y}[27]$.

В начальный момент времени спиновая система находится в состоянии термодинамического равновесия. Квантовые корреляции удобно изучать после возбуждения системы резонансными (по спинам $I$ и $S$ ) 90-градусными высокочастотными импульсами магнитного поля, приложенными вдоль осей $y$ BCK [27]. После возбуждающего 
действия импульсов матрица плотности системы имеет вид

$$
\begin{aligned}
\rho_{0} & =\frac{1}{Z} e^{\beta\left(\omega_{A} I_{x}+\omega_{B} S_{x}\right)}= \\
& =\frac{1}{Z}\left(\operatorname{ch}^{2} \frac{\beta \omega_{A}}{2}+I_{x} \operatorname{sh} \beta \omega_{A}+4 I_{1 x} I_{2 x} \operatorname{sh}^{2} \frac{\beta \omega_{A}}{2}\right)\left(\operatorname{ch} \frac{\beta \omega_{B}}{2}+2 S_{x} \operatorname{sh} \frac{\beta \omega_{B}}{2}\right),
\end{aligned}
$$

где $\beta$ пропорциональна обратной температуре системы, $\omega_{A}$ и $\omega_{B}$ - ларморовы частоты и $Z=8 \operatorname{ch}^{2}\left(\beta \omega_{A} / 2\right) \operatorname{ch}\left(\beta \omega_{B} / 2\right)$ - статистическая сумма.

Структура гамильтониана гетероядерных ДДВ (1) позволяет заключить, что гамильтониан $H$ коммутирует с гамильтонианом секулярных ДДВ спинов подсистемы $A$. Это означает, что только подсистема $A$ подвержена гомоядерной спиновой дипольной эволюции. Поскольку унитарные локальные преобразования не изменяют квантовых корреляций (квантового дискорда) [2], мы не будем учитывать ДДВ спинов подсистемы $A$. Таким образом, эволюция матрицы плотности (2) определяется только гамильтонианом гетероядерных ДДВ (1).

В ходе эволюции системы, определяемой гамильтонианом $H(1)$, матрица плотности системы $\rho(t)$ принимает форму

$$
\begin{aligned}
\rho(t)= & e^{-i H t} \rho_{0} e^{i H t}=\frac{1}{Z}\left\{\left(A+S_{z} B\right) \operatorname{ch} \frac{\beta \omega_{B}}{2}+\right. \\
& +S_{x} \operatorname{sh} \frac{\beta \omega_{B}}{2}\left[2 A\left(\cos ^{2} \frac{g t}{2}-4 I_{1 z} I_{2 z} \sin ^{2} \frac{g t}{2}\right)-i B I_{z} \sin g t\right]+ \\
& \left.+S_{y} \operatorname{sh} \frac{\beta \omega_{B}}{2}\left[2 A I_{z} \sin g t+i B\left(\cos ^{2} \frac{g t}{2}-4 I_{1 z} I_{2 z} \sin ^{2} \frac{g t}{2}\right)\right]\right\},
\end{aligned}
$$

где операторы $A$ и $B$ имеют следующий вид:

$$
\begin{aligned}
& A=\operatorname{ch}^{2} \frac{\beta \omega_{A}}{2}+I_{x} \operatorname{sh} \beta \omega_{A} \cos \frac{g t}{2}+\operatorname{sh}^{2} \frac{\beta \omega_{A}}{2}\left(2 I_{x}^{2} \cos ^{2} \frac{g t}{2}+2 I_{y}^{2} \sin ^{2} \frac{g t}{2}-1\right), \\
& B=2 I_{y} \operatorname{sh} \beta \omega_{A} \sin \frac{g t}{2}-i\left[\left(I^{+}\right)^{2}-\left(I^{-}\right)^{2}\right] \operatorname{sh}^{2} \frac{\beta \omega_{A}}{2} \sin g t
\end{aligned}
$$

a $I^{+}$и $I^{-}-$повышающий и понижающий операторы углового спинового момента.

Для определения меры квантовых корреляций - квантового дискорда - нам потребуются редуцированные по подсистемам $A$ и $B$ матрицы плотности

$$
\begin{aligned}
& \rho_{A}(t)=\operatorname{Tr}_{B} \rho(t)=\frac{2}{Z} A \operatorname{ch} \frac{\beta \omega_{B}}{2}, \\
& \rho_{B}(t)=\operatorname{Tr}_{A} \rho(t)=\frac{1}{2}+S_{x} \text { th } \frac{\beta \omega_{B}}{2} \cos ^{2} \frac{g t}{2} .
\end{aligned}
$$




\section{3. МИНИМИЗАЦИЯ КВАНТОВОЙ УСЛОВНОЙ ЭНТРОПИИ В ВЫСОКОТЕМПЕРАТУРНОМ ПРИБЛИЖЕНИИ}

Согласно современным представлениям [29] полные корреляции в двухсоставной системе определяются взаимной информацией $I(\rho)$,

$$
I(\rho)=S\left(\rho_{A}\right)+S\left(\rho_{B}\right)-S(\rho),
$$

где энтропия системы находится по формуле

$$
\begin{aligned}
S(\rho)= & 3+2 \log _{2} \operatorname{ch} \frac{\beta \omega_{A}}{2}+\log _{2} \operatorname{ch} \frac{\beta \omega_{B}}{2}- \\
& -\log _{2}\left(e^{\beta \omega_{A}}\right) \text { th } \frac{\beta \omega_{A}}{2}-\frac{1}{2} \log _{2}\left(e^{\beta \omega_{B}}\right) \text { th } \frac{\beta \omega_{B}}{2} .
\end{aligned}
$$

Энтропии подсистем вычисляются с помощью редуцированных матриц плотности (6), (7). Для вычисления квантового дискорда нам потребуется энтропия подсистемы $B$, которая равна

$$
\begin{aligned}
S\left(\rho_{B}\right)=1-\frac{1}{2}[(1 & \left.+ \text { th } \frac{\beta \omega_{B}}{2} \cos ^{2} \frac{g t}{2}\right) \log _{2}\left(1+\text { th } \frac{\beta \omega_{B}}{2} \cos ^{2} \frac{g t}{2}\right)+ \\
& \left.+\left(1-\operatorname{th} \frac{\beta \omega_{B}}{2} \cos ^{2} \frac{g t}{2}\right) \log _{2}\left(1-\operatorname{th} \frac{\beta \omega_{B}}{2} \cos ^{2} \frac{g t}{2}\right)\right],
\end{aligned}
$$

а для вычисления классических корреляций необходима энтропия подсистемы $A$

$$
S\left(\rho_{A}\right)=-\sum_{i=1}^{4} \mu_{i} \log _{2} \mu_{i}
$$

где $\mu_{i}(i=1,2,3,4)$ - собственные числа матрицы $\rho_{A}(t)(11)$, которые равны

$$
\begin{aligned}
\mu_{1} & =\frac{1}{4}\left(1-\operatorname{th}^{2} \frac{\beta \omega_{A}}{2} \cos g t\right), \\
\mu_{2} & =\frac{1}{4} \operatorname{ch}^{-2} \frac{\beta \omega_{A}}{2}, \\
\mu_{3,4} & =\frac{1}{4}\left(1+\operatorname{th}^{2} \frac{\beta \omega_{A}}{2} \cos ^{2} \frac{g t}{2} \pm \operatorname{th} \frac{\beta \omega_{A}}{2} \sqrt{\operatorname{th}^{2} \frac{\beta \omega_{A}}{2} \sin ^{2} \frac{g t}{2}+4 \cos ^{2} \frac{g t}{2}}\right) .
\end{aligned}
$$

Взаимная квантовая информация может быть определена другим способом [2], [3]. Для этого необходимо провести на одной из подсистем (в настоящей работе - на подсистеме $B$ ) полный набор ортогональных проективных измерений

$$
\left\{C_{k}=V \Pi_{k} V^{\dagger}: V \in S U(2), \Pi_{k}=|k\rangle\langle k|, k=0,1\right\} .
$$

Данная формула показывает, что таких наборов измерений может быть сколь угодно много, и каждый из них задается унитарной матрицей $V$, которая может быть представлена следующим образом:

$$
V=t I+i \vec{y} \vec{\sigma}
$$


где $I$ - единичная матрица, $t \in \mathbb{R}, \vec{y}=\left(y_{1}, y_{2}, y_{3}\right) \in \mathbb{R}^{3}$, а $\vec{\sigma}=\left(\sigma_{x}, \sigma_{y}, \sigma_{z}\right)$ - матрицы Паули $\left(\sigma_{\alpha}=2 S_{\alpha}, \alpha=x, y, z\right)$. Из унитарности матрицы $V$ вытекает соотношение

$$
t^{2}+y_{1}^{2}+y_{2}^{2}+y_{3}^{2}=1 \text {. }
$$

Для дальнейшего анализа удобно ввести параметры [14]

$$
z_{1}=2\left(-t y_{2}+y_{1} y_{3}\right), \quad z_{2}=2\left(t y_{1}+y_{2} y_{3}\right), \quad z_{3}=t^{2}-y_{1}^{2}-y_{2}^{2}+y_{3}^{2} .
$$

Из соотношений (15) и (16) следует, что

$$
z_{1}^{2}+z_{2}^{2}+z_{3}^{2}=1
$$

После проведения измерений $C_{k}$ на подсистеме $B$ матрица плотности системы $\rho$ изменится. Мы получим ансамбль состояний $\left\{p_{k}, \rho_{k}\right\}(k=0,1)$, в котором

$$
p_{k}=\operatorname{Tr}\left\{\left(I_{A} \otimes C_{k}\right) \rho\left(I_{A} \otimes C_{k}\right)\right\}, \quad \rho_{k}=\frac{1}{p_{k}}\left(I_{A} \otimes C_{k}\right) \rho\left(I_{A} \otimes C_{k}\right) .
$$

В этих выражениях $I_{A}$ - единичный оператор размера $4 \times 4$, действующий на подсистему $A$. Условная энтропия $S\left(\rho \mid\left\{C_{k}\right\}\right)$ системы после проведенных измерений определяется по формуле [14], [15]

$$
S\left(\rho \mid\left\{C_{k}\right\}\right)=p_{0} S\left(\rho_{0}\right)+p_{1} S\left(\rho_{1}\right),
$$

где $S\left(\rho_{k}\right)$ - энтропия состояния с матрицей плотности $\rho_{k}(k=0,1)$.

Условная энтропия системы $S\left(\rho \mid\left\{C_{k}\right\}\right)$ зависит от использованного набора измерений. При этом взаимная информация определяется по формуле

$$
I^{\prime}(\rho)=S\left(\rho_{A}\right)-S\left(\rho \mid\left\{C_{k}\right\}\right)
$$

В классических системах формулы (8) и (20) ведут к одинаковому результату, однако в квантовых системах из-за особенностей квантово-механических измерений это может быть не так [2], [3]. Различие в результатах, получаемых с помощью формул (8) и (20), может рассматриваться как мера "квантовости" изучаемой системы. При этом необходимо избавиться от произвола в определении величины квантовой условной энтропии из-за произвольности полного набора ортогональных проективных измерений подсистемы $B$, зависящего от унитарных преобразований $\left\{C_{k}\right\}$. Исходя из того что полная классическая часть корреляций является максимальным количеством информации о подсистеме $A$, которую можно извлечь с помощью измерений на подсистеме $B$ [30], в работе [3] было предложено взять в качестве меры чисто классических корреляций величину

$$
\tilde{I}(\rho)=S\left(\rho_{A}\right)-\min _{\left\{C_{k}\right\}} S\left(\rho \mid\left\{C_{k}\right\}\right),
$$

где минимизация осуществляется по всем наборам $\left\{C_{k}\right\}$ унитарных преобразований. Мерой квантовых корреляций тогда является дискорд $D$, определяемый следующим образом [2]:

$$
D=I(\rho)-\tilde{I}(\rho)
$$


После измерений (13) в рассматриваемом случае система может быть описана [25] ансамблем состояний $\left\{p_{k}, \rho_{k}\right\}(k=0,1)$ с населенностями

$$
p_{0}=\frac{1}{2}+\frac{z_{1}}{2} \operatorname{th} \frac{\beta \omega_{B}}{2} \cos ^{2} \frac{g t}{2}, \quad p_{1}=\frac{1}{2}-\frac{z_{1}}{2} \text { th } \frac{\beta \omega_{B}}{2} \cos ^{2} \frac{g t}{2}
$$

и матрицами плотности

$$
\begin{aligned}
\rho_{k}= & \frac{2}{Z\left(1 \pm z_{1} \operatorname{th}\left(\beta \omega_{B} / 2\right) \cos ^{2}(g t / 2)\right)}\left\{\left(A \pm \frac{z_{3}}{2} B\right) \operatorname{ch} \frac{\beta \omega_{B}}{2} \pm\right. \\
& \pm \frac{z_{1}}{2} \operatorname{sh} \frac{\beta \omega_{B}}{2}\left[2 A\left(\cos ^{2} \frac{g t}{2}-4 I_{1 z} I_{2 z} \sin ^{2} \frac{g t}{2}\right)-i B I_{z} \sin g t\right] \pm \\
& \left. \pm \frac{z_{2}}{2} \operatorname{sh} \frac{\beta \omega_{B}}{2}\left[2 A I_{z} \sin g t+i B\left(\cos ^{2} \frac{g t}{2}-4 I_{1 z} I_{2 z} \sin ^{2} \frac{g t}{2}\right)\right]\right\}, \quad k=0,1,
\end{aligned}
$$

где параметры $z_{1}, z_{2}, z_{3}$ описывают различные унитарные преобразования $\left\{C_{k}\right\}$, ответственные за проведение измерений на подсистеме $B$ [14], [15]. Эти параметры будут использованы ниже для минимизации условной энтропии $S\left(\rho \mid\left\{C_{k}\right\}\right)$ системы после измерений в подсистеме $B$.

Минимизацию условной энтропии при произвольных температурах аналитическими методами вряд ли возможно провести. Однако это удается сделать в высокотемпературном приближении [27], когда

$$
x=\frac{1}{2} \beta \omega_{B}<1, \quad y=\frac{1}{2} \beta \omega_{A}<1 .
$$

Вычисление условной энтропии (19) с помощью формул (3)-(7), (23), (24) с точностью до членов порядка $x^{2}$ и $y^{2}$ включительно приводит к формуле

$$
\begin{aligned}
S\left(\rho \mid\left\{C_{k}\right\}\right)=- & \frac{1}{8 \ln 2}\left\{4 z_{1}^{2}\left(x^{2} \sin ^{4} \tau-2 y^{2} \sin ^{2} \tau\right)+\right. \\
& \left.+2 z_{2}^{2}\left(x^{2} \sin ^{2} 2 \tau-4 y^{2} \sin ^{2} \tau\right)+a(x, y)\right\}
\end{aligned}
$$

где

$$
a(x, y)=8 y^{2}-8 \ln 2 \cdot\left(2+x^{2}+2 y^{2}\right)
$$

а безразмерное время $\tau=g t / 2$. Таким образом, задача сводится к минимизации функции

$$
f\left(z_{1}, z_{2}\right)=-4 z_{1}^{2}\left(x^{2} \sin ^{4} \tau-2 y^{2} \sin ^{2} \tau\right)-2 z_{2}^{2}\left(x^{2} \sin ^{2} 2 \tau-4 y^{2} \sin ^{2} \tau\right)
$$

при условии $z_{1}^{2}+z_{2}^{2} \leqslant 1$, поскольку $z_{1}^{2}+z_{2}^{2}+z_{3}^{2}=1$ [14], [15]. 
ТАБлицА 1. Значения параметров $z_{1}, z_{2}, z_{3}$, при которых условная квантовая энтропия $S\left(\rho \mid\left\{C_{k}\right\}\right)$ достигает минимума, для различных соотношений между величинами $x$ и $y$ в процессе эволюции системы на временном интервале $0 \leqslant \tau \leqslant \pi / 2$.

\begin{tabular}{|c|c|c|c|c|c|c|c|c|c|c|c|}
\hline & & -1 & \multicolumn{9}{|c|}{$\tau \in\left(\arccos \gamma_{1}, \pi / 2\right)$} \\
\hline 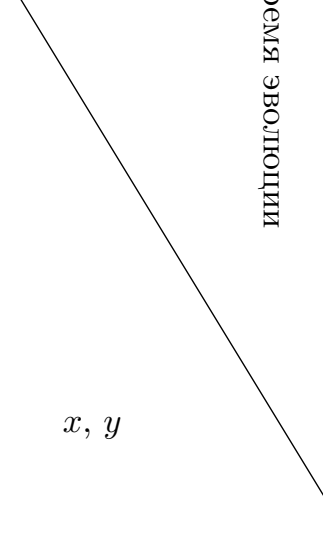 & $\begin{array}{l}\text { Oे } \\
0 \\
0 \\
0 \\
\delta \\
己 \\
己 \\
己\end{array}$ & 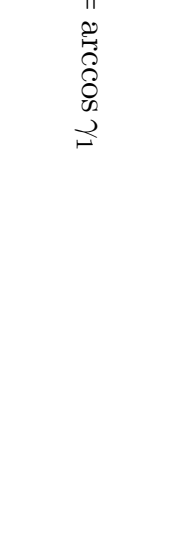 & 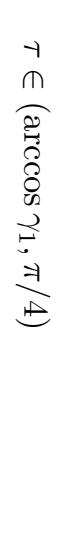 & $\begin{array}{l}-1 \\
\| \\
\Rightarrow \\
\not\end{array}$ & 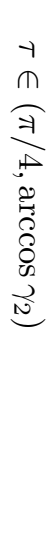 & 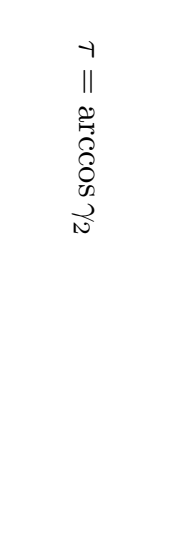 & 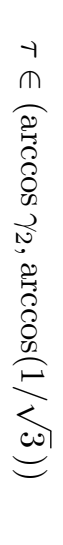 & 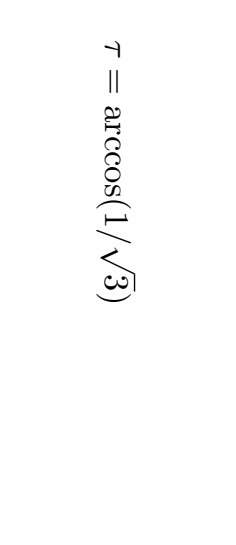 & 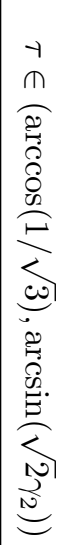 & 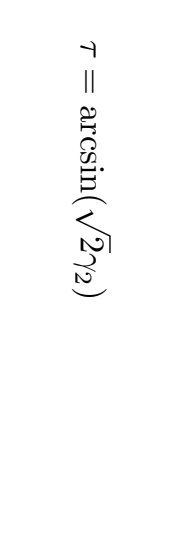 & 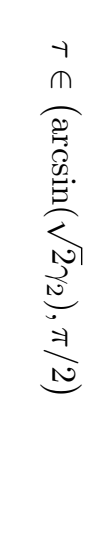 \\
\hline$y \geqslant x$ & \multicolumn{11}{|c|}{$z_{3}^{2}=1$} \\
\hline $\begin{array}{c}y=\gamma_{1} x \\
\gamma_{1} \in(1 / \sqrt{2}, 1)\end{array}$ & $z_{2}^{2}=1$ & $z_{2}^{2}+z_{3}^{2}=1$ & \multicolumn{9}{|c|}{$z_{3}^{2}=1$} \\
\hline$y=x / \sqrt{2}$ & & $z_{2}^{2}=1$ & & $z_{2}^{2}+z_{3}^{2}=1$ & & & & $z_{3}^{2}=1$ & & & \\
\hline $\begin{array}{c}y=\gamma_{2} x \\
\gamma_{1} \in(1 / \sqrt{3}, 1 / \sqrt{2})\end{array}$ & \multicolumn{5}{|c|}{$z_{2}^{2}=1$} & $z_{2}^{2}+z_{3}^{2}=1$ & \multicolumn{3}{|c|}{$z_{3}^{2}=1$} & $z_{1}^{2}+z_{3}^{2}=1$ & $z_{1}^{2}=1$ \\
\hline$y=x / \sqrt{3}$ & \multicolumn{7}{|c|}{$z_{2}^{2}=1$} & $z_{1}^{2}+z_{2}^{2}+z_{3}^{2}=1$ & \multicolumn{3}{|c|}{$z_{1}^{2}=1$} \\
\hline$y<x / \sqrt{3}$ & \multicolumn{7}{|c|}{$z_{2}^{2}=1$} & $z_{1}^{2}+z_{2}^{2}=1$ & \multicolumn{3}{|c|}{$z_{1}^{2}=1$} \\
\hline
\end{tabular}


Условный минимум функции $f\left(z_{1}, z_{2}\right)$ зависит от соотношения между параметрами $x$ и $y$, пропорциональными ларморовским частотам, а также от безразмерного времени эволюции $\tau$. Для нахождения минимума функции $f\left(z_{1}, z_{2}\right)$ необходимо определять знаки выражений в скобках в формуле (28) при различных соотношениях между параметрами $x$ и $y$ и безразмерным временем $\tau$. Эта задача сводится к решению системы элементарных неравенств. Найденные параметры $z_{1}, z_{2}, z_{3}$, при которых условная энтропия $S\left(\rho \mid\left\{C_{k}\right\}\right)$ системы после измерений на подсистеме $B$ достигает минимального значения в ходе эволюции, представлены в табл. 1 . Во многих случаях минимальное значение условной энтропии достигается, когда один из параметров $z_{i}(i=1,2,3)$ равен по модулю единице, а два других равны нулю. Однако в определенные моменты эволюции системы коэффициенты при $z_{1}$ и $z_{2}$ в формуле (28) совпадают, и тогда единице равняется сумма квадратов двух параметров, а третий параметр равен нулю. И, наконец, может случиться ситуация, когда минимальное значение условной энтропии вообще не зависит от параметров $z_{i}$ $(i=1,2,3)$, т. е. $z_{1}^{2}+z_{2}^{2}+z_{3}^{2}=1$.

Если $\omega_{A} \gg \omega_{B}$, то основными корреляциями между подсистемами $A$ и $B$ являются $z z$-корреляции, что соответствует минимальному значению условной энтропии при $\left|z_{3}\right|=1$. Проведенные вычисления показывают (см. табл. 1), что этот вывод справедлив в ходе всей эволюции системы при $y>x$, т. е. при $\omega_{A}>\omega_{B}$.

Рассмотрим теперь противоположный случай ${ }^{1)}$, когда $\omega_{A}=0$. В подсистеме $A$ тогда нет поляризации, и не возникает намагниченность после 90-градусного импульса. Спину подсистемы $B$ просто нечего вращать. Корреляция возникает только из-за того, что спины подсистемы $A$ вращают спин подсистемы $B$ в плоскости $x y$ после 90-градусного высокочастотного импульса. За такие корреляции ответственны параметры $z_{1}$ и $z_{2}$. Табл. 1 показывает, что $x y$-корреляции в основном преобладают в системе при $x \geqslant y$, т. е. при $\omega_{B} \geqslant \omega_{A}$.

На рис. 2 представлен график зависимости квантового дискорда от времени эволюции при высоких температурах, когда $x=0.015, y=0.03$. График симметричен относительно $\tau=\pi / 2$, что соответствует проведенному теоретическому анализу (см. также формулу (31) ниже). Отметим, что величина дискорда при высоких температурах мала.

\section{4. КВАНТОВЫЙ ДИСКОРД И КВАНТОВЫЕ КОРРЕЛЯЦИИ ПРИ НИЗКИХ ТЕМПЕРАТУРАХ}

При низких температурах, когда $x>1$ и $y>1$ и высокотемпературное приближение, использованное в предыдущем разделе, неприменимо, исследование минимума условной энтропии $S\left(\rho \mid\left\{C_{k}\right\}\right)$ можно провести только численными методами. Проведенные нами расчеты показали, что минимальные значения условной энтропии снова получаются при таких же соотношениях между параметрами $z_{1}, z_{2}, z_{3}$, как и в высокотемпературном приближении. Однако найти области значений ларморовских частот и моментов времени эволюции системы, когда искомые параметры $z_{i}(i=1,2,3)$ обеспечивают минимум условной энтропии, здесь затруднительно. На

\footnotetext{
1) На этот случай наше внимание обратил В. Е. Зобов.
} 


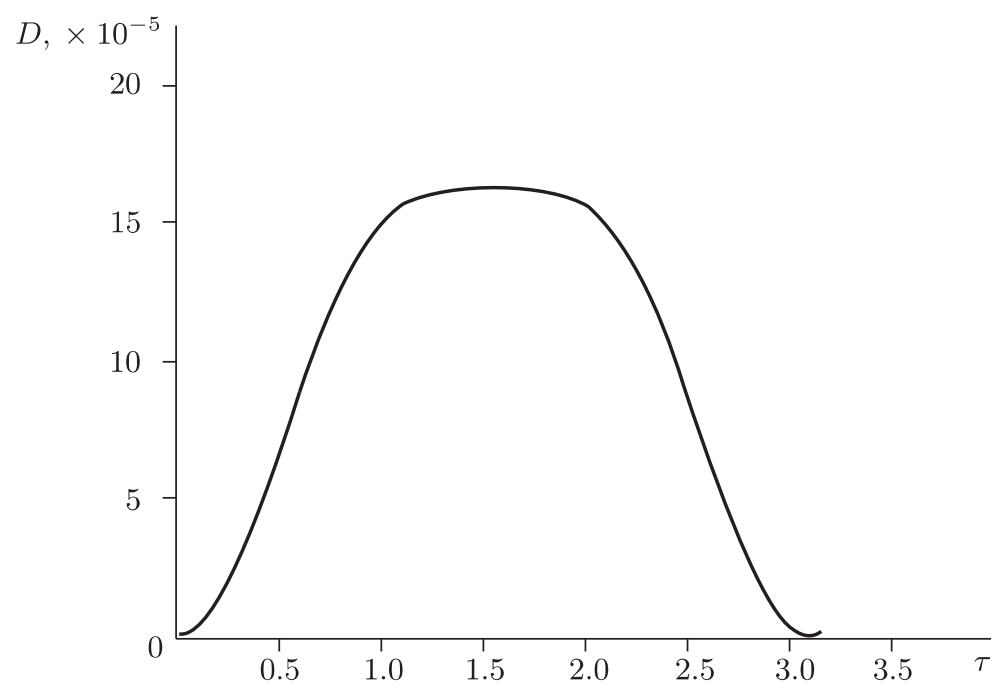

Рис. 2. Квантовый дискорд при высоких температурах $(x=0.015$, $y=0.03)$, когда глобальный минимум условной энтропии $S\left(\rho \mid\left\{C_{k}\right\}\right)=1.9987$ реализуется при $z_{1}=z_{2}=0$ (при этом $\left|z_{3}\right|=1$ ) и не зависит от времени $\tau$ (только для случая $y>x$, см. текст после формулы (30)).

рис. 3 приведена условная энтропия $S\left(\rho \mid\left\{C_{k}\right\}\right)$ при различных значениях параметров $z_{1}, z_{3}$ (в области $\left|z_{1}\right| \leqslant 1,\left|z_{3}\right| \leqslant 1$ ) для $x=3, y=1.5$ и $\tau=1.112$. Помимо глобального минимума при $\left|z_{2}\right|=1$, условная энтропия имеет минимумы и при $\left|z_{1}\right|=1$, что затрудняет аналитическое исследование задачи.

Проведенные нами численные расчеты при $y>x$ показали, что в этом случае минимальное значение квантовой условной энтропии достигается при $\left|z_{3}\right|=1$ как при высоких, так и при низких температурах для любого времени эволюции. При $z_{1}=z_{2}=0$ матрицы плотности $\rho_{k}(k=0,1)$, заданные в $(24)$, упрощаются и принимают вид

$$
\rho_{k}=\frac{2}{Z}\left(A \pm \frac{B}{2}\right) \operatorname{ch} x, \quad k=0,1 .
$$

Выполняя унитарное преобразование

$$
\tilde{\rho}_{k}=e^{i(-1)^{k} \tau I_{z}} \rho_{k} e^{-i(-1)^{k} \tau I_{z}}
$$

с помощью формул (4), (5) устанавливаем, что матрицы $\tilde{\rho}_{k}(k=0,1)$ не зависят от времени. Последнее означает, что собственные числа матриц $\rho_{k}$, определяющих минимальную условную энтропию, также не зависят от времени. Таким образом, в рассматриваемом случае условная квантовая энтропия $S\left(\rho \mid\left\{C_{k}\right\}\right)$ не зависит от 


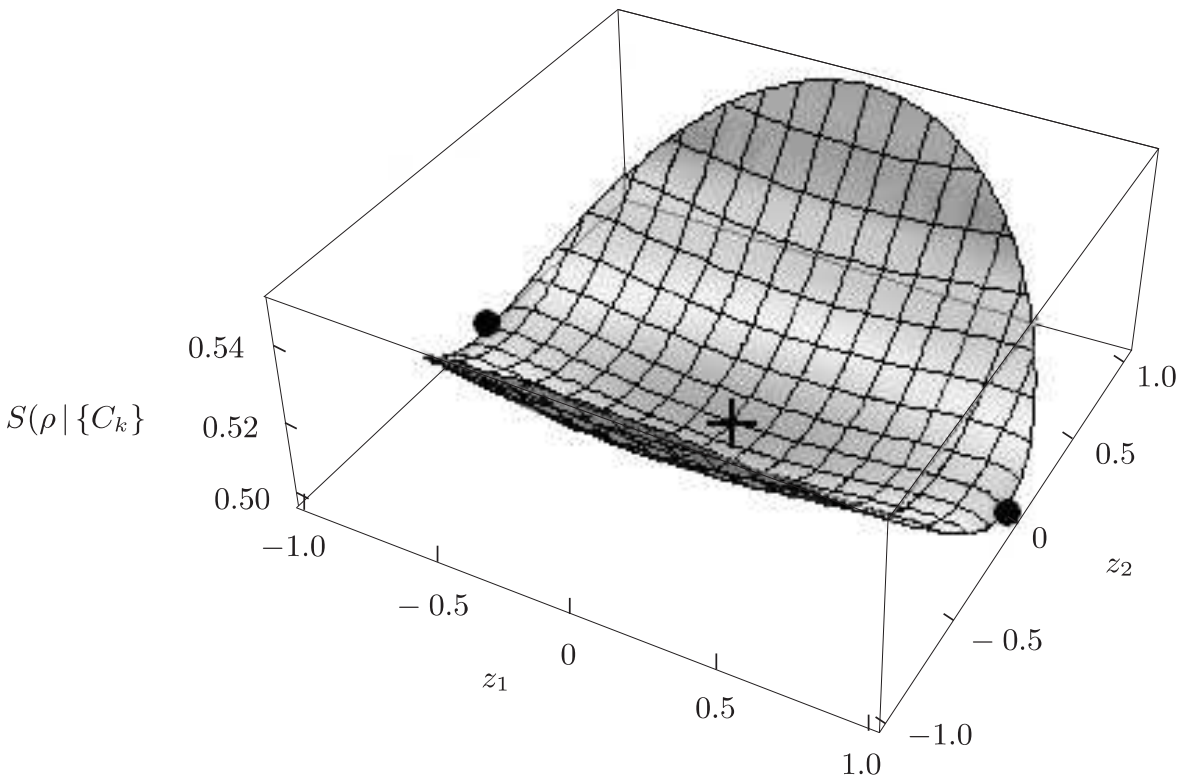

Рис. 3. Условная энтропия $S\left(\rho \mid\left\{C_{k}\right\}\right)$ при $x=3, y=1.5$ в момент времени $\tau=1.112$. Крестиком обозначен глобальный минимум $S\left(\rho \mid\left\{C_{k}\right\}\right)=0.497268$, соответствующий значениям $z_{1}=z_{3}=0$ (при этом $\left|z_{2}\right|=1$ ), точками показаны два локальных минимума $S\left(\rho \mid\left\{C_{k}\right\}\right)=0.498854$, соответствующих $z_{2}=z_{3}=0$ (при этом $\left|z_{1}\right|=1$ ).

времени в процессе эволюции системы. Квантовый дискорд $D$ (при $\left|z_{3}\right|=1$ ) вычисляется теперь с помощью формул (4), (5), (8)-(10), (21)-(23), (19), (29) и равен

$$
\begin{aligned}
D= & -\frac{1}{2}\left[\left(1+\operatorname{th} x \cos ^{2} \tau\right) \log _{2}\left(1+\operatorname{th} x \cos ^{2} \tau\right)+\left(1-\operatorname{th} x \cos ^{2} \tau\right) \log _{2}\left(1-\text { th } x \cos ^{2} \tau\right)\right]- \\
& -\log _{2} \operatorname{ch} x+\log _{2}\left(e^{x}\right) \operatorname{th} x .
\end{aligned}
$$

Эта формула показывает, что квантовый дискорд $D$ не зависит от параметра $y$, т. е. от большей ларморовской частоты $\omega_{A}\left(\omega_{A}>\omega_{B}\right)$. Если $x=0$ (т. е. $\omega_{B}=0$ ), то дискорд (31) обращается в ноль. В этом случае $z z$-корреляции в системе отсутствуют. И, наконец, отметим, что, по крайней мере в высокотемпературном приближении, в двухсоставной трехспиновой системе во внешнем магнитном поле для случая $\omega_{A}>\omega_{B}$ мы получили аналитическое выражение для квантового дискорда.

Вычисление квантового дискорда для других случаев, когда минимальное значение квантовой условной энтропии получается при других значениях параметров $z_{1}, z_{2}, z_{3}$, оказывается значительно более трудоемкой задачей. В качестве примера рассмотрим случай, когда минимальное значение квантовой условной энтропии достигается при $\left|z_{2}\right|=1, z_{1}=z_{3}=0$. В этом случае собственные числа $\nu_{k i}$ матриц $\rho_{k}$ 
$(k=0,1$ и $i=1,2,3,4)$ могут быть представлены в виде

$$
\nu_{k i}=\frac{1}{4}+\frac{2}{Z} \lambda_{k i}
$$

где $\lambda_{k i}(k=0,1$ и $i=1,2,3,4)$ удовлетворяют уравнению четвертой степени

$$
\begin{aligned}
\lambda_{k i}^{4} & -\lambda_{k i}^{2}\left[\bar{a}^{2} \operatorname{sh}^{2} x \sin ^{2} 2 \tau+\left(\bar{c}^{2}+\bar{d}^{2}\right) \operatorname{ch}^{2} x+2\left(u_{k}^{2}+v_{k}^{2}\right)\right]+ \\
& +\lambda_{k i}\left[ \pm 2 \bar{a}\left(v_{k}^{2}-u_{k}^{2}\right) \operatorname{sh} x \sin 2 \tau-2 \bar{d}\left(u_{k}^{2}+v_{k}^{2}\right) \operatorname{ch} x-4 \bar{c} u_{k} v_{k} \operatorname{ch} x\right]+ \\
& +\bar{a}^{2} \bar{d}^{2} \operatorname{ch}^{2} x \operatorname{sh}^{2} x \sin ^{2} 2 \tau \pm 2 \bar{a} \bar{d}\left(v_{k}^{2}-u_{k}^{2}\right) \operatorname{ch} x \operatorname{sh} x \sin 2 \tau- \\
& -4 \bar{c} \bar{d} u_{k} v_{k} \operatorname{ch}^{2} x+\bar{c}^{2} \bar{d}^{2} \operatorname{ch}^{4} x=0,
\end{aligned}
$$

где

$$
\bar{a}=\operatorname{ch}^{2} y, \quad \bar{c}=\operatorname{sh}^{2} y \cos 2 \tau, \quad \bar{d}=\operatorname{sh}^{2} y,
$$

а зависящие от индекса $k$ величины $u_{k}$ и $v_{k}$ равны

$$
\begin{aligned}
& u_{k}=\frac{1}{2} \operatorname{sh} 2 y \cdot(\operatorname{ch} x \cos \tau \pm \operatorname{sh} x \sin \tau), \\
& v_{k}=\frac{1}{2} \operatorname{sh} 2 y \cdot(\operatorname{ch} x \cos \tau \mp \operatorname{sh} x \sin \tau) .
\end{aligned}
$$

Один из корней $\lambda_{1}$ уравнения (33), не зависящий от индекса $k$, можно найти в явном виде,

$$
\lambda_{1}=-d \operatorname{ch} x,
$$

а три других корня удовлетворяют кубическому уравнению

$$
\begin{aligned}
\lambda_{k i}^{3} & -\lambda_{k i}^{2} \bar{d} \operatorname{ch} x-\lambda_{k i}\left[\bar{a}^{2} \operatorname{sh}^{2} x \sin ^{2} 2 \tau+\bar{c}^{2} \operatorname{ch}^{2} x+2\left(u_{k}^{2}+v_{k}^{2}\right)\right] \pm \\
& \pm 2 \bar{a}\left(v_{k}^{2}-u_{k}^{2}\right) \operatorname{sh} x \sin 2 \tau-4 \bar{c} u_{k} v_{k} \operatorname{ch} x+ \\
& +\bar{a}^{2} \bar{d} \operatorname{ch} x \operatorname{sh}^{2} x \sin ^{2} 2 \tau+\bar{c}^{2} \bar{d} \operatorname{ch}^{3} x=0, \quad k=0,1, \quad i=2,3,4 .
\end{aligned}
$$

Как и следовало ожидать, собственные числа $\lambda_{k i}(k=0,1$ и $i=2,3,4)$ зависят от обеих ларморовых частот $\omega_{A}$ и $\omega_{B}$, в отличие от предыдущего случая. Существенно также, что собственные числа $\lambda_{k i}$ являются функциями времени эволюции системы $\tau$. Поэтому условная квантовая энтропия $S\left(\rho \mid\left\{C_{k}\right\}\right)$ здесь зависит от времени в ходе эволюции системы. Используя формулы $(21),(22)$, для квантового дискорда можно получить следующее выражение:

$$
\begin{aligned}
D=-2-\frac{1}{2}\left[\left(1+\operatorname{th} x \cos ^{2} \tau\right) \log _{2}\left(1+\text { th } x \cos ^{2} \tau\right)+\right. \\
\left.+\left(1-\operatorname{th} x \cos ^{2} \tau\right) \log _{2}\left(1-\operatorname{th} x \cos ^{2} \tau\right)\right]- \\
-\log _{2}\left(\operatorname{ch}^{2} y \operatorname{ch} x\right)+\log _{2}\left(e^{y}\right) \operatorname{th} y+\frac{1}{2} \log _{2}\left(e^{x}\right) \operatorname{th} x- \\
-p_{0} \sum_{i=1}^{4} \nu_{0 i} \log _{2} \nu_{0 i}-p_{1} \sum_{i=1}^{4} \nu_{1 i} \log _{2} \nu_{1 i},
\end{aligned}
$$



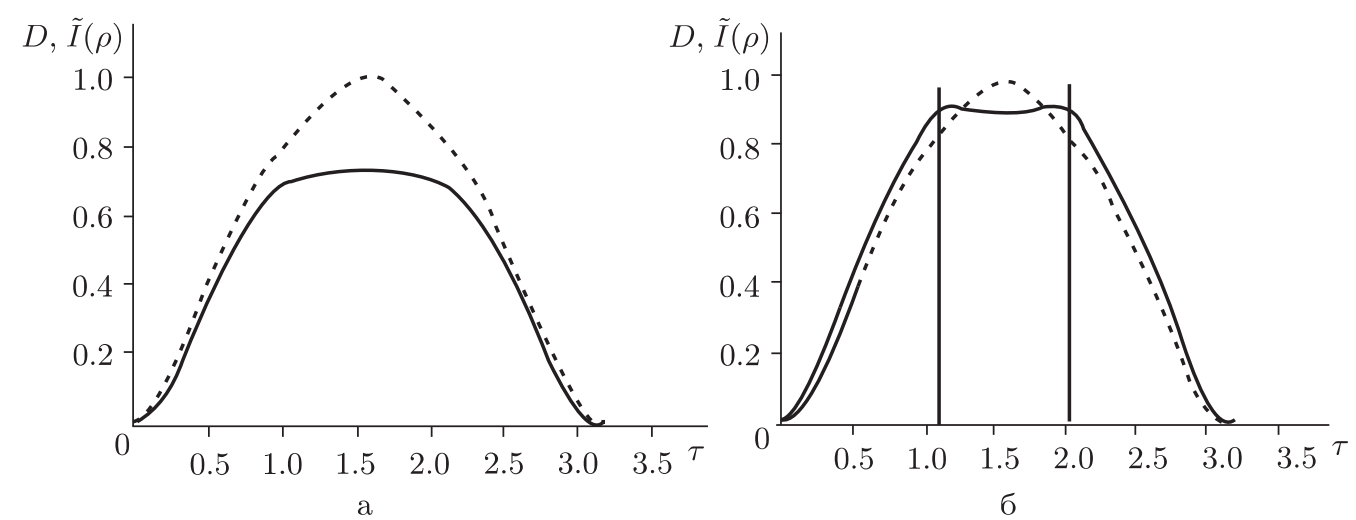

Рис. 4. Классические корреляции $\tilde{I}(\rho)$ (штриховые линии) и квантовые корреляции $D$ (сплошные линии) при эволюции системы в двух случаях: когда минимум условной энтропии лежит в $z_{1}=z_{2}=0,\left|z_{3}\right|=1$ при $x=1.5$ и $y=3(\mathrm{a})$, и когда минимум достигается при $z_{1}=z_{3}=0,\left|z_{2}\right|=1$ на интервалах $\tau \in(0,1.1167)$ и $\tau \in(2.0246, \pi)$, а на интервале $\tau \in(1.1167,2.0246)$ он достигается в точке $z_{2}=z_{3}=0,\left|z_{1}\right|=1$ при $x=3$ и $y=1.5$ (б).

где населенности $p_{0}, p_{1}$ определяются по формуле $(23)$, а $\nu_{k i}(k=0,1$ и $i=1,2,3,4)$ по формуле (32).

Минимальная условная энтропия связана с классическими корреляциями (см. формулу (21)) [3], в то время как квантовый дискорд ответствен за квантовые корреляции [2]. Мы сравнили классические и квантовые корреляции при эволюции системы как в случае минимума условной энтропии при $z_{1}=z_{2}=0,\left|z_{3}\right|=1$ (рис. 4a) при $x=1.5$ и $y=3$, так и в более сложной ситуации (рис. 4б) для $x=3$ и $y=1.5$. В этом случае минимум достигается при $\left|z_{2}\right|=1$ на интервалах $\tau \in(0,1.1167)$ и $\tau \in(2.0246, \pi)$, а на интервале $\tau \in(1.1167,2.0246)$ - при $\left|z_{1}\right|=1$. В первом случае (рис. 4a), когда преобладают $z z$-корреляции, классические корреляции превосходят квантовые в ходе всей эволюции системы. Во втором случае (рис. 4б), когда определяющими являются $x x$-корреляции, наоборот, квантовые корреляции преобладают над классическими на большей части временного интервала. Однако в интервале $\tau \in(1.2742,1.8634)$ преобладают классические корреляции.

\section{5. ЗАКЛЮЧЕНИЕ}

Мы рассмотрели двухсоставную трехспиновую систему во внешнем магнитном поле при произвольной температуре. В высокотемпературном случае [27] нам удалось аналитически найти минимум условной квантовой энтропии при произвольных значениях ларморовских частот подсистем и в любой момент времени эволюции системы. В результате впервые удалось получить аналитические формулы для квантового дискорда в двухсоставной трехкубитной системе как для случая преобладающих $z z$-корреляций, так и для случая $x y$-корреляций.

Мы также получили аналитические выражения для квантового дискорда при низких температурах без определения точных границ областей ларморовских частот, для которых справедливы найденные формулы. Мы исследовали эволюцию 
квантового дискорда и соотношения классических и квантовых корреляций для различных наборов параметров $z_{1}, z_{2}, z_{3}$, при которых достигается минимум условной энтропии.

Благодарности. Авторы выражают искреннюю благодарность В. Е. Зобову за стимулирующие обсуждения. Работа поддержана РФФИ (гранты № 13-03-00017 и 13-03-12418) и Программой Президиума РАН № 8 "Разработка методов получения химических веществ и создание новых материалов".

\section{Список литературы}

[1] М. Нильсен, И. Чанг, Квантовые вычисления и квантовая информация, Мир, М., 2006.

[2] H. Ollivier, W. H. Zurek, Phys. Rev. Lett., 88:1 (2001), 017901, 4 pp.

[3] L. Henderson, V. Vedral, J. Phys. A, 34:35 (2001), 6899-6905.

[4] K. Modi, A pedagogical overview of quantum discord, arXiv: 1312.7676.

[5] A. Datta, A. Shaji, C. M. Caves, Phys. Rev. Lett., 100:5 (2008), 050502, 4 pp.

[6] A. Datta, A. Shaji, Int. J. Quantum Inf., 9:07-08 (2011), 1787-1805.

[7] S. Pirandola, Quantum discord as a resource for quantum cryptography, arXiv: 1309.2446.

[8] J. A. Jones, S.D. Karlen, J. Fitzsimons, A. Ardavan, S. C. Benjamin, G. A. D. Briggs, J. J. L. Morton, Science, 324:5931 (2009), 1166-1168.

[9] S. Simmons, J. A. Jones, S.D. Karlen, A. Ardavan, J. J. L. Morton, Phys. Rev. A, 82:2 (2010), 022330, 5 pp.

[10] K. Modi, H. Cable, M. Williamson, V. Vedral, Phys. Rev. X, 1:2 (2011), 021022, 9 pp.

[11] W.S. Warren, Science, 294:5546 (2001), 1475-1476.

[12] H. Katiyar, S. S. Roy, T. S. Mahesh, A. Patel, Phys. Rev. A, 86:1 (2012), 012309, 8 pp.

[13] I. S. Oliveira, T. J. Bonagamba, R. S. Sarthour, J. C. C. Freitas, E. R. deAzevedo, NMR Quantum Information Processing, Elsevier, Amsterdam, 2007.

[14] S. Luo, Phys. Rev. A, 77:4 (2008), 042303, 6 pp.

[15] M. Ali, A. R. P. Rau, G. Alber, Phys. Rev. A, 81:4 (2010), 042105, 7 pp.

[16] Y. Huang, Phys. Rev. A, 88:1 (2013), 014302, 3 pp.

[17] C. C. Rulli, M. S. Sarandy, Phys. Rev. A, 84:4 (2011), 042109, 6 pp.

[18] Шунь-Лун Ло, Шуан-Шуан Фу, ТМФ, 171:3 (2012), 519-528.

[19] В. Е. Зобов, ТМФ, 177:1 (2013), 111-125.

[20] B. Dakic, V. Vedral, C. Brukner, Phys. Rev. Lett., 105:19 (2010), 190502, 4 pp.

[21] B. Bellomo, R. Lo Franco, G. Compagno, Phys. Rev. A, 86:1 (2012), 012312, 6 pp.

[22] M. Piani, Phys. Rev. A, 86:3 (2012), 034101, 3 pp.

[23] E. B. Fel'dman, E. I. Kuznetsova, M. A. Yurishchev, J. Phys. A: Math. Theor., 45:47 (2012), 475304, $11 \mathrm{pp}$.

[24] V.E. Zobov, Proc. SPIE, 8700 (2012), 87001D, 10 pp.

[25] A. Y. Chernyavskiy, S. I. Doronin, E. B. Fel'dman, Phys. Scr., T160 (2014), 014007, 5 pp.

[26] А. Ю. Чернявский, Микроэлектроника, 38:3 (2009), 217-223.

[27] М. Гольдман, Спиновая температура и ядерный магнитный резонанс в твердых телах, Мир, М., 1972.

[28] K. Sato, S. Nakazawa, R. Rahimi et al., J. Mater. Chem., 19:22 (2009), 3739-3754.

[29] V. Vedral, Introduction to Quantum Information Science, Oxford Univ. Press., Oxford, 2008.

[30] С. М. Алдошин, Э. Б. Фельдман, М. А. Юрищев, ФНТ, 40:1 (2014), 5-21.

Поступила в редакцию 13.03.2014, после доработки 29.05.2014 